\title{
Chemoattractant-mediated leukocyte trafficking enables HIV dissemination from the genital mucosa
}

\author{
Maud Deruaz, ${ }^{1}$ Thomas T. Murooka, ${ }^{1}$ Sophina Ji, ${ }^{1}$ Marc A. Gavin, ${ }^{2}$ Vladimir D. Vrbanac, ${ }^{1}$ \\ Judy Lieberman, ${ }^{3}$ Andrew M. Tager, ${ }^{1}$ Thorsten R. Mempel, ${ }^{1}$ and Andrew D. Luster ${ }^{1}$ \\ ${ }^{1}$ Center for Immunology and Inflammatory Diseases, Division of Rheumatology, Allergy and Immunology, Massachusetts \\ General Hospital, Harvard Medical School, Boston, Massachusetts, USA. ${ }^{2}$ Amgen Inc., Thousand Oaks, California, USA. \\ ${ }^{3}$ Program in Cellular and Molecular Medicine, Boston Children's Hospital, Harvard Medical School, Boston, \\ Massachusetts, USA.
}

HIV vaginal transmission accounts for the majority of newly acquired heterosexual infections. However, the mechanism by which HIV spreads from the initial site of viral entry at the mucosal surface of the female genital tract to establish a systemic infection of lymphoid and peripheral tissues is not known. Once the virus exits the mucosa it rapidly spreads to all tissues, leading to $\mathrm{CD}^{+} \mathrm{T}$ cell depletion and the establishment of a viral reservoir that cannot be eliminated with current treatments. Understanding the molecular and cellular requirements for viral dissemination from the genital tract is therefore of great importance, as it could reveal new strategies to lengthen the window of opportunity to target the virus at its entry site in the mucosa where it is the most vulnerable and thus prevent systemic infection. Using HIV vaginal infection of humanized mice as a model of heterosexual transmission, we demonstrate that blocking the ability of leukocytes to respond to chemoattractants prevented HIV from leaving the female genital tract. Furthermore, blocking lymphocyte egress from lymph nodes prevented viremia and infection of the gut. Leukocyte trafficking therefore plays a major role in viral dissemination, and targeting the chemoattractant molecules involved can prevent the establishment of a systemic infection.

Conflict of interest: The authors have declared that no conflict of interest exists.

Submitted: May 12, 2016

Accepted: February 16, 2017

Published: April 6, 2017

Reference information: JCI Insight. 2017;2(7):e88533. https:// doi.org/10.1172/jci.insight.88533.

\section{Introduction}

Heterosexual transmission of HIV to women accounts for the majority of newly acquired infections (1). The early events following HIV vaginal exposure necessary to establish a systemic infection are not known, as they are not easily studied in humans. Investigators have turned to SIV infection of nonhuman primates (NHPs) to model viral transmission and dissemination. These studies suggest that following acquisition and initial replication in the female genital tract (FGT), SIV reaches the lymph nodes (LNs) where viral replication takes off due to readily available target cells and then rapidly spreads to the gut and other organs before being detected in the blood (2). A similar pattern of viral dissemination was recently described following SIV infection of the male genital tract (3). By analogy, a similar pattern of dissemination has been attributed to HIV infection following vaginal acquisition (4). However, the kinetics, pattern, and mechanism of HIV spread from the initial site of viral entry at the mucosal surface of the FGT to establish a systemic infection of lymphoid and peripheral tissue are not known. Understanding the mechanisms of HIV spread in vivo will inform new therapeutic interventions to interrupt viral dissemination and provide a greater opportunity to eliminate the virus in the FGT where it is most vulnerable and prevent a systemic infection.

Since cell-to-cell transmission of HIV is more efficient than free virus-to-cell transmission in vitro (5), HIV might therefore also disseminate more efficiently in vivo in infected $\mathrm{CD} 4^{+} \mathrm{T}$ cells or carried by $\mathrm{DCs}$ or macrophages (6). However, the role of leukocyte trafficking in viral dissemination following intravaginal (IVAG) infection, a natural route of infection, has not been demonstrated. Leukocyte entry into and exit from tissues are tightly regulated processes controlled by trafficking molecules, including chemoattractants. Interfering with leukocyte trafficking could therefore be a new approach to prevent the spread of HIV beyond the FGT and the establishment of a systemic infection and lengthen the window of opportunity to target infection. The chemokine receptor CCR7 directs T cell and DC egress from peripheral tissues into afferent lymphatics and LNs at homeostasis (7-9). The sphingosine 1-phosphate receptor 1 (S1PR1) 
A

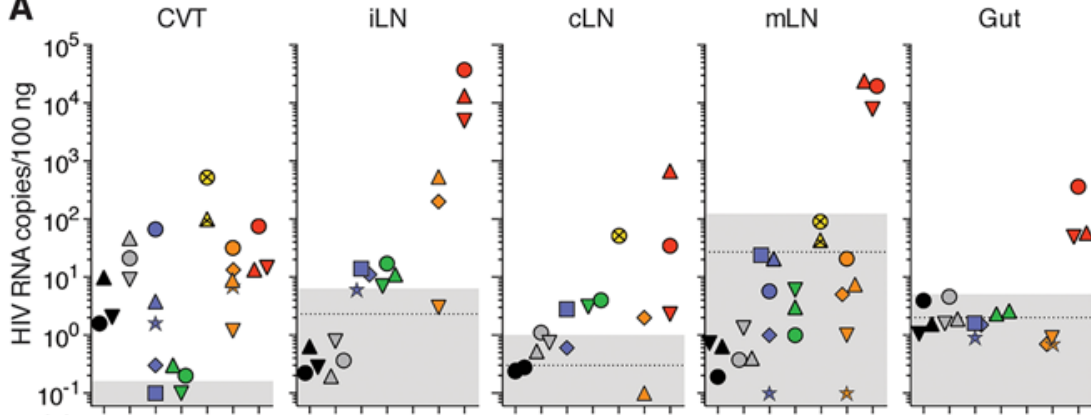

dpi $\begin{array}{lllllllllllllllllllllllllllllllllllll}0.751 & 2 & 4 & 6 & 8 & 14 & 0.751 & 2 & 4 & 6 & 8 & 14 & 0.75 & 2 & 4 & 6 & 8 & 14 & 0.75 & 2 & 2 & 4 & 6 & 8 & 14 & 0.75 & 2 & 4 & 6 & 8 & 14\end{array}$
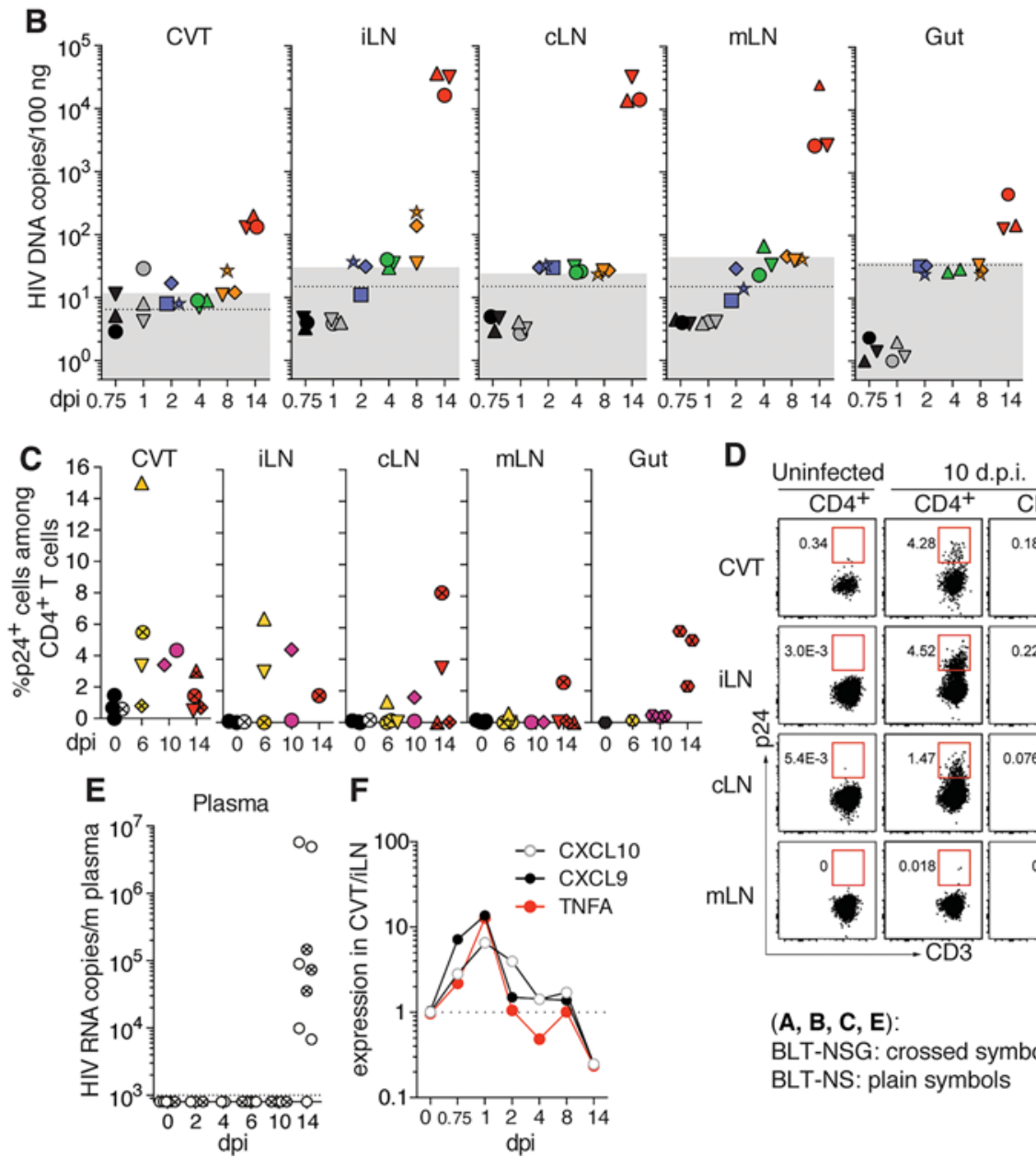

Gut

\section{D}

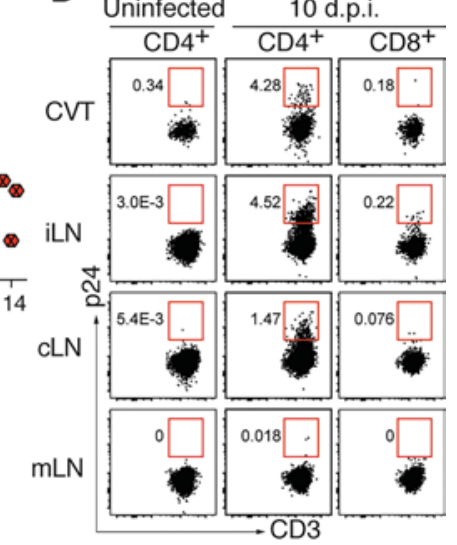

(A, B, C, E):

BLT-NSG: crossed symbols BLT-NS: plain symbols
Figure 1. HIV dissemination in humanized BLT mice following intravaginal (IVAG) infection. BLT-NOD-scid (NS, plain symbols) and BLT-NODscid IL2R $\gamma^{-1-}$ (NSG, crossed symbols) mice were sacrificed at different days postinfection (dpi) with $1 \times 10^{5} 50 \%$ tissue culture infectious dose $\left(\mathrm{TCID}_{50}\right)$ R5-tropic HIV strain JRCSF (HIV JRCSF $_{\text {) }}$ IVAC ( $n=2-4$ per time point). The presence of (A) viral RNA by RT-qPCR, (B) DNA by qPCR, or (C) infected T cells by p24+ flow cytometry, was analyzed in different tissues. Mice from which tissues were harvested on the same dpi are shown with the same color and each mouse within one color group is represented by a different symbol. Dotted line: mean value of tissue from 3 to 5 uninfected mice; gray box: \pm 2 SD of mean value of uninfected mice. (D) Representative flow cytometry plots showing the percentage of HIV p24+ human $\mathrm{CD} 4^{+} \mathrm{T}$ cells in tissues of uninfected (left panels) and infected (middle panels) BLT-NS mice 10 dpi. Human CD8 ${ }^{+} T$ cells (right panels) were used as negative controls. (E) HIV RNA in plasma detected by RT-qPCR. Dotted line: limit of sensitivity of the assay corresponding to 3 copies/ $\mu$ I HIV RNA ( $n$ = 2-7 per time point). (F) Ratio of human TNFA, CXCL9, and CXCL10 expression in CVT compared to iLNs at different dpi as measured by qPCR on tissue cDNA using a mean of 3 animals per group. CVT, cervico-vaginal tissue; LNs, lymph nodes; iLNs, iliac LNs; cLNs, cervical LNs; mLNs, mesenteric LNs.

has also been implicated in this process, particularly in inflammation $(10,11)$. We therefore hypothesized that CCR7 and S1PR1 might play a role in the spread of HIV from the FGT to the lymphoid compartment and peripheral tissue.

The reconstitution of mucosal tissues with human immune cells in humanized bone marrow/liver/thymus (BLT) mice allows for productive IVAG HIV transmission (12-15). In addition, the cross-reactivity of homing molecules between mice and humans is sufficient to allow for the study of human immune cell trafficking in these

mice (16). BLT mice can therefore be used as a surrogate in vivo model of HIV IVAG infection (12-16) to study the earliest events following viral acquisition and analyze the mechanism of viral dissemination from the cervico-vaginal tissue (CVT). Using these mice, we have established the kinetics of viral dissemination in vivo following HIV IVAG infection, and addressed whether leukocyte trafficking mediated by chemoattractant receptors in general, and CCR7 and S1PR1 in particular, are required for HIV dissemination from the FGT to establish systemic infection.

\section{Results}

HIV dissemination in BLT mice following IVAG infection. Humanized BLT mice were challenged with a single atraumatic IVAG application of $1 \times 10^{5} 50 \%$ tissue culture infectious dose $\left(\mathrm{TCID}_{50}\right.$ ) R5-tropic HIV strain JRCSF $\left(\mathrm{HIV}_{\text {JRCSF }}\right)$ to obtain an infection rate of $96 \%$ (Supplemental Figure $1 \mathrm{~A}$; supplemental material available online with this article; https://doi.org/10.1172/jci.insight.88533DS1) and to allow synchronized 
A
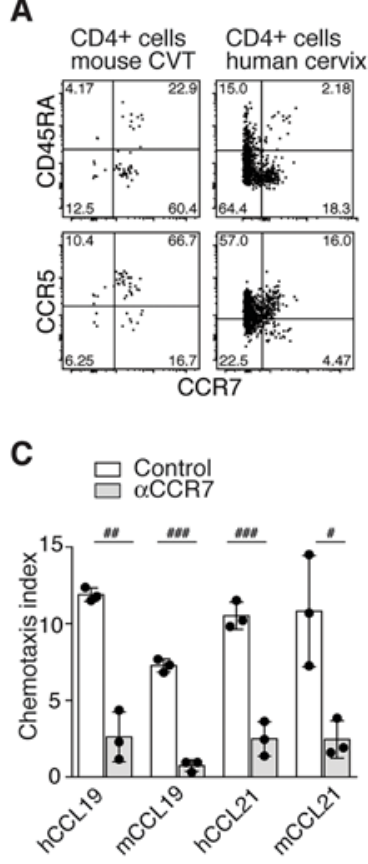

B

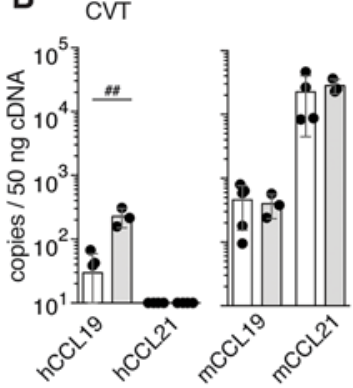

D

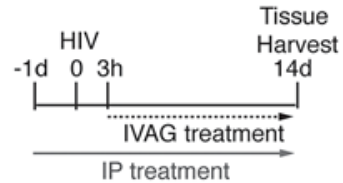

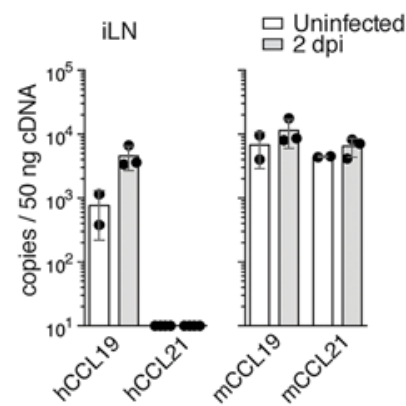

E Peripheral Bloo

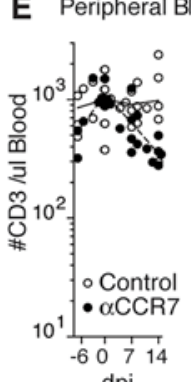

dpi

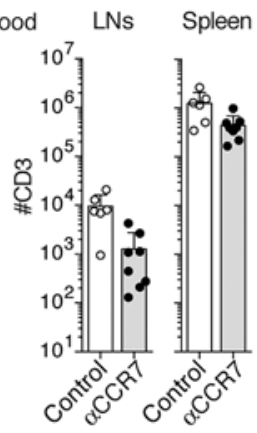

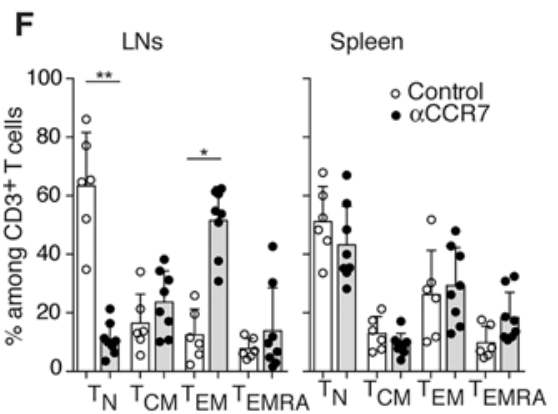
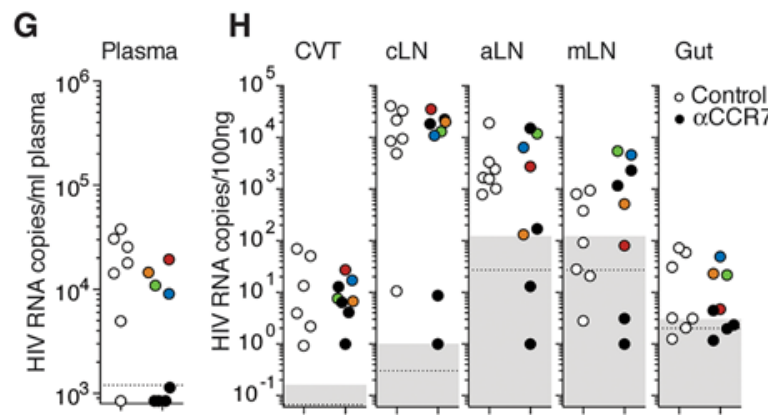

Figure 2. CCR7 inhibition did not block HIV dissemination from CVT. (A) Representative flow cytometry plots of 2 experiments showing the percentage of CD4 ${ }^{+}$T cells expressing CD45RA and CCR7 (top panels) and CCR5 and CCR7 (bottom panels) isolated from the CVT of BLT-NSC (left panels) and from human cervical tissue (right panels). (B) Human and murine CCL19 and CCL21 expression in CVT and iLNs in uninfected (open bars, $n=3$ ) and infected (gray bars, $n=3$ ) BLT-NS mice 2 days postinfection (dpi) as measured by qPCR on tissue cDNA. (C) In vitro chemotaxis assay of human peripheral blood T cells in response to human or murine CCL19 and CCL21 in the presence of CCR7 mAb ( $\alpha$ CCR7, gray bars, $n=3$ ) or isotype control (open bars, $n=3$ ). In $\mathbf{B}$ and $\mathbf{C}$ each dot represents 1 mouse and bars represent mean \pm SD. ${ }^{\#} P<0.05,{ }^{\#} P<0.01$, ${ }^{\# \#} P<0.001$ by Student's $t$ test. (D) Scheme of in vivo experimental treatment. BLT-NSG mice received human $\gamma$-globulins (hlgG) and murine Fc receptor-blocking mAb (2.4G2) with PBS (control, $n=7$ ) or CCR7 mAb ( $\alpha$ CCR7, $n=8$ ) i.p. every third day starting 1 day prior to intravaginal (IVAG) HIV. CCR7 mAb-treated mice received CCR7 mAb IVAG starting 3 hours postinfection (hpi) and control mice received PBS. (E) Numbers of human CD3+ $T$ cells in peripheral blood, LNs, and spleen. (F) Percentage of naive $\left(T_{N}\right.$ : $\left.C D 45 R A+C D 62 L^{+}\right)$, central memory $\left(T_{C M}\right.$ : $\left.C D 45 R A-C D 62 L^{+}\right)$, effector memory $\left(T_{E M}\right.$ : $\left.C D 45 R A^{-} C D 62 L^{-}\right)$and effector memory reexpressing CD45RA ( $T_{\text {EMRA }}$ : CD45RA ${ }^{+} \mathrm{CD}$ 62 $\mathrm{L}^{-}$) $\mathrm{T}$ cells in $\mathrm{LNs}$ and spleen of control-treated (open circles) or CCR7 mAb-treated (closed circles) mice. Each circle in $\mathbf{E}$ and $\mathbf{F}$ represents 1 mouse and bars represent mean $+\mathrm{SD} .{ }^{*} P<0.05,{ }^{*} P<0.01$ by Kruskal-Wallis test and Fisher exact test. Presence of HIV RNA in plasma (G) and tissues $(\mathbf{H})$ at $14 \mathrm{dpi}$ of control or CCR7 mAb-treated mice. Results corresponding to CCR7 mAb-treated mice with positive viremia are shown with different colors. Dotted line: limit of sensitivity of the assay; gray box: \pm 2 SD of mean value of 3 to 5 uninfected animals. CVT, cervico-vaginal tissue; LNs, lymph nodes; cLNs, cervical; aLNs, axillary LNs; mLNs, mesenteric LNs.

infections between mice. These experiments were performed using both BLT-NOD-scid (NS) and BLTNOD-scid IL2R $\gamma^{-1-}$ (NSG) mice. The presence of viral RNA (vRNA) and DNA (vDNA) was analyzed in tissues to determine the kinetics of HIV dissemination (Figure 1, A and B). At 18 hours postinfection (hpi) and 24 hpi, vRNA was detected only in CVT (Figure 1A). vDNA was first detected in the CVT at 1 day postinfection (dpi) (Figure 1B), suggesting that vRNA present at $18 \mathrm{hpi} \mathrm{might} \mathrm{be} \mathrm{from} \mathrm{the} \mathrm{inoculum.}$ At 2 to 8 dpi, vRNA was detected in the draining iliac LNs (iLNs) as well as the cervical LNs (cLNs) (Figure 1A). However, vRNA was first detected in the mesenteric LNs (mLNs) and gut (small and large intestine) at $14 \mathrm{dpi}$. Similar kinetics were observed for the presence of infected cells by vDNA analyses (Figure 1B). The presence of productively infected cells was confirmed by intracellular staining for HIV 
A

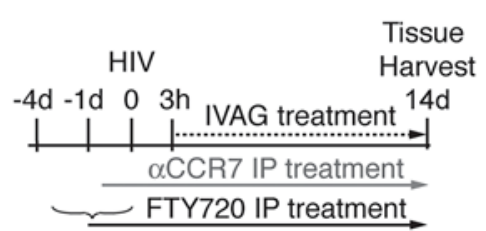

B

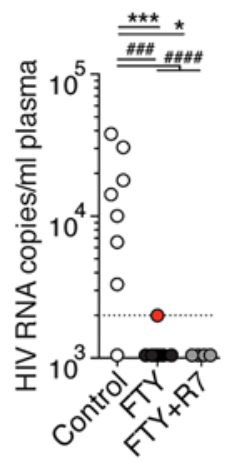

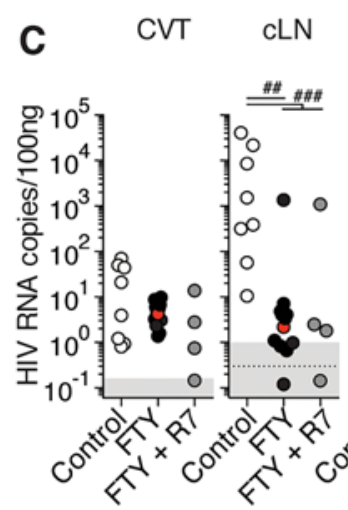
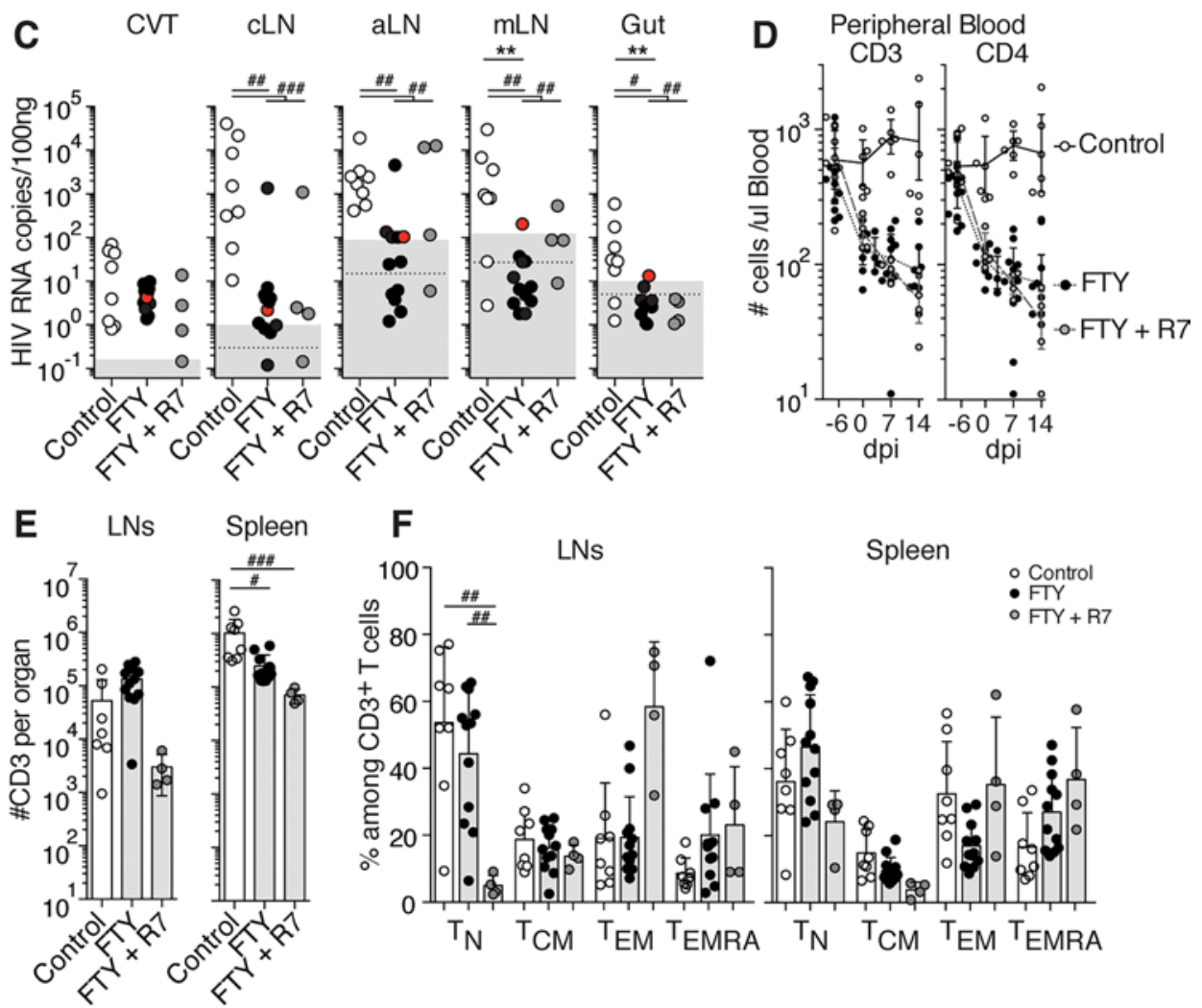

Figure 3. FTY720 treatment interferes with HIV spread to the lymph nodes (LNs) and prevents infection from leaving LNs. (A) Scheme of the experimental treatment. BLT-NSG mice received PBS (control) or FTY720 daily i.p. starting 4 days prior to or on the day of intravaginal (IVAG) infection, and received PBS or FTY720 daily IVAG starting 3 hours postinfection. CCR7 mAb ( $\alpha$ CCR7) i.p. and IVAG treatments were performed as described in Figure 2. Presence of HIV RNA in plasma (B) and tissues (C) at 14 days postinfection (dpi) of control (open circles, $n=8$ ), FTY720-treated (FTY, black circles, $n=12$ ), and FTY720+CCR7 mAb-treated (FTY+R7, gray circles, $n=4)$ mice. Each circle in $\mathbf{B}-\mathbf{F}$ represents 1 mouse. The FTY720-treated mouse with borderline viremia is highlighted in red. Dotted line: limit of sensitivity of the assay; gray box: \pm 2 SD of the mean of 3 to 5 uninfected animals. (D) Numbers of human $\mathrm{CD}^{+}$and $\mathrm{CD}^{+} \mathrm{T}$ cells in peripheral blood, (E) numbers of human $\mathrm{CD3}^{+}$in $\mathrm{LNs}$ and spleen, and (F) percentages of $\mathrm{T}_{N}, \mathrm{~T}_{\mathrm{CM}}$, $T_{E M}, T_{E M R A}$ in LNs and spleen of control, FTY-treated, and FTY+R7-treated mice. Bars in $\mathbf{E}$ and $\mathbf{F}$ represent mean + SD. CVT, cervico-vaginal tissue; cLNs, cervical LNs; aLNs, axillary LNs; $\mathrm{mLNs}$, mesenteric LNs; $T_{N}$, naive $T$ cells; $T_{C M}$, central memory $T$ cells; $T_{\text {EM }}$, effector memory $T$ cells; $T_{\text {EMRA }}$, effector memory $T$ cells reexpressing CD45RA. Kruskal-Wallis test was used to perform statistical comparisons of mean percentages and viral load $(\# P<0.05$, \#\# $P$

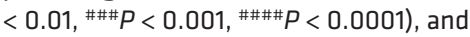
Fisher exact test was used to perform statistical comparisons of the number of viremic and nonviremic tissues in treated versus untreated mice $\left({ }^{*} P<0.05,{ }^{* *} P<\right.$ $0.01,{ }^{* * *} P<0.001$ ).

p24 (Figure 1, C and D). At 6 and 10 dpi, the percentage of CD4+ T cells that were HIV p24 $4^{+}$was higher in the CVT and iLNs than in cLNs. Infected cells were also first detected in mLNs and the gut at $14 \mathrm{dpi}$. Finally, vRNA was not detectable in plasma (Figure 1E) or in spleen (data not shown) before 14 dpi, which coincided with a pronounced increase in vRNA and vDNA in all LNs and infection of the gut. Consistent with these kinetics, human cytokine and chemokine responses were first detected in the CVT as early as 18 to $24 \mathrm{hpi}$, which were then followed by responses in iLNs beginning at 2 dpi (Figure $1 \mathrm{~F}$, and Supplemental Figure 2, A and B). In addition, a redistribution of human $\mathrm{CD} 4^{+}$and $\mathrm{CD} 8^{+} \mathrm{T}$ cells was observed in the CVT at $2 \mathrm{dpi}$, with the formation of cellular aggregates located below the epithelium (Supplemental Figure 2, C and D). These data demonstrate that HIV disseminates from the CVT to the lymphoid compartment in humanized mice before spreading to the gut and being detectable in plasma. While BLT-NS showed higher plasma viral loads at 14 dpi (Supplemental Figure 1A), similar viral loads were obtained in tissues in BLT-NS compared to BLT-NSG (Supplemental Figure 1B). Furthermore, no differences in the pattern or kinetics of viral dissemination to the different LNs or gut were observed between BLT-NS and BLT-NSG mice (Figure 1), even though BLT-NS mice have better reconstitution of some peripheral LNs (Supplemental Figure 1D). However, BLT-NSG mice had higher percentages of human lymphocytes in peripheral blood and higher numbers of human $\mathrm{CD}^{+} \mathrm{T}$ cells in gut lamina propria than BLT-NS mice (Supplemental Figure 1, C and D). In addition, we have found that BLT-NSG 
A

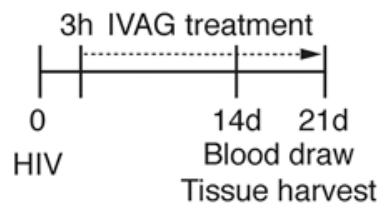

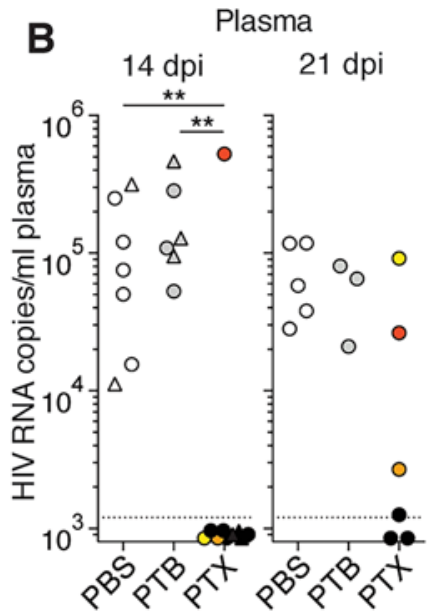

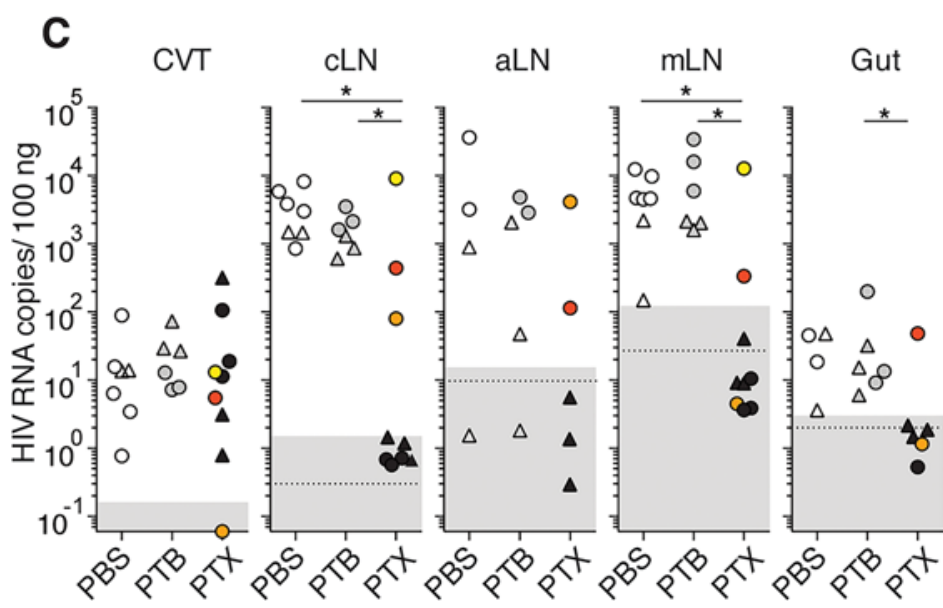

Figure 4. Pertussis toxin (PTX) treatment delays HIV dissemination from the CVT. (A) Scheme of the experimental procedure. Daily intravaginal (IVAG) treatment with PBS, pertussis toxin oligomer $B$ (PTB), or PTX was started 3 hours after IVAG HIV. (B) Presence of HIV RNA in plasma at 14 and 21 days postinfection (dpi) of PBS-treated (open symbols, $n=7$ ), PTX-treated (black symbols, $n=9$ ), or PTBtreated (gray symbols, $n=6$ ) BLT-NSG mice. Mice were sacrificed at 14 (triangles) or 21 (circles) dpi. One mouse with positive viremia at $14 \mathrm{dpi}$ is shown in red, and 2 mice with positive viremia at $21 \mathrm{dpi}$ are shown in orange and yellow. (C) HIV RNA in tissue at 14 (triangles) or 21 (circles) dpi. Tissues corresponding to the 3 mice with positive viremia are shown as in B. Of note, for some of the aLN samples the amount and quality of RNA was insufficient for analysis and gut samples from 3 PBS- and 3 PTX-treated mice were not collected. Dotted line: limit of sensitivity of the assay; gray box: mean of 3 to 5 uninfected animals \pm 2 SD. CVT, cervico-vaginal tissue; LNs, lymph nodes; cLNs, cervical LNs; aLNs, axillary LNs; mLNs, mesenteric LNs. Fisher exact tests were used to perform statistical comparisons of the number of viremic and nonviremic tissues in treated versus untreated mice. ${ }^{*} P<0.05,{ }^{* *} P<0.01$.

mice are more consistently reconstituted in less time than BLT-NS mice, allowing for the generation of larger batches of BLT-NSG mice per tissue donor. All subsequent experiments were therefore performed using BLT-NSG mice.

CCR7-dependent leukocyte trafficking is not critical for HIV dissemination. We assessed the role of CCR7 in HIV dissemination from the CVT. The majority of human $\mathrm{CD} 4^{+} \mathrm{T}$ cells present in the FGT of BLT mice are of a central memory phenotype, a subset that expresses CCR7 (Figure 2A and ref. 15). The majority of these CCR $7^{+} \mathrm{CD} 4^{+} \mathrm{T}$ cells in the CVT are also positive for CCR5 and therefore potential HIV target cells. A corresponding CCR $7^{+} \mathrm{CCR} 5^{+} \mathrm{CD} 4^{+} \mathrm{T}$ cell population was found in human cervical tissue (Figure 2A). In humanized mice, murine CCR7 ligands (CCL19 and CCL21) are expressed at higher levels than the human ligands in the CVT and iLNs, as might be expected since stromal cells are the main source of these chemokines (Figure 2B). Importantly, murine CCL19 and CCL21 are able to activate CCR7 on human T cells (Figure 2C).

To block CCR7-mediated immune cell trafficking, humanized mice were treated with an antihuman CCR7 IgG1 monoclonal antibody (CCR7 mAb) devoid of N-linked glycosylation (Supplemental Figure 3). This $\mathrm{mAb}$ was designed to impair $\mathrm{Fc}_{\mathrm{c}}$ receptor binding and antibody-dependent cellular cytotoxicity (17), while preserving CCR7 blocking capabilities (Figure 2C). However, as early as 24 hpi, $\mathrm{T}$ cell depletion was observed in peripheral blood and tissues of CCR7 mAb-treated mice (Supplemental Figures 4 and 5). Humanized BLT mice have impaired B cell maturation and, as a result, have low levels of endogenous IgG compared with human and mouse plasma (ref. 18 and Supplemental Figure 5A). We hypothesized that CCR7 mAb-induced $\mathrm{T}$ cell depletion might be due to a lack of endogenous IgG to compete with the exogenous CCR7 mAb for binding of Fc receptors. Consistent with this hypothesis, coadministration of pooled human IgG (hIgG) and a murine Fc receptor-blocking $\mathrm{mAb}\left(2.4 \mathrm{G} 2\right.$ ) prevented the CCR7 $\mathrm{mAb}$ treatment from depleting human $\mathrm{CD}^{+} \mathrm{T}$ cells (Supplemental Figure 5, B-D).

To specifically block CCR7-mediated immune trafficking, humanized mice were therefore treated with hIgG and 2.4G2 with or without CCR7 mAb (Figure 2D). Human T cell numbers in peripheral blood and spleen remained comparable in CCR7 mAb-treated and control mice (Figure 2E). Lower numbers of $\mathrm{T}$ cells were present in LNs of CCR7 mAb-treated mice (Figure 2E and Supplemental Figure 5D), with decreased percentages of naive $\mathrm{T}$ cells $\left(\mathrm{CD} 45 \mathrm{RA}^{+} \mathrm{CD} 62 \mathrm{~L}^{+}\right.$) (Figure $2 \mathrm{~F}$ and Supplemental Figure 6). This is consistent with CCR7 $\mathrm{mAb}$ treatment interfering with human naive T cell trafficking to LNs from the blood via high endothelial venules in humanized mice, as it does for murine $\mathrm{T}$ cells in conventional mice (9). Furthermore, we found that following CCR7 mAb treatment, CCR7 was not detected on human 
T cells present in tissues and blood by subsequent CCR7 staining (Supplemental Figures 6 and 7), demonstrating CCR7 blockade of immune cells in tissue.

We compared HIV viral loads in the plasma and tissues of PBS- and CCR7 mAb-treated mice at 14 dpi (Figure 2, G and H). All mice in the PBS-treated group had positive viremia, whereas of the 8 mice in the CCR7 mAb-treated group, 4 were positive, 1 was at the limit of detection, and 3 were negative (Figure $2 \mathrm{G}$ ). Mice from both groups had similar levels of virus in the CVT (Figure 2H), demonstrating that the treatment did not interfere with the acquisition of a local infection. All mice showing positive or borderline viremia were also positive in all LNs and the gut. Of the 3 mice with negative viremia, only 1 was also negative in all LNs and gut, whereas the 2 other mice were positive in the cLNs but not in other tissues (Figure $2 \mathrm{H}$ ). Of note, iLNs in BLT-NSG mice were too small to be analyzed. Analysis of vDNA showed similar results (Supplemental Figure 8). Treatment with CCR7 mAb therefore did not significantly delay HIV dissemination from the CVT to the LNs.

S1PR1-mediated immune cell trafficking participates in HIV dissemination. As S1PR1 has been described to participate in the regulation of $\mathrm{T}$ cell and DC egress from peripheral tissues to LNs during inflammation $(10,11,19)$, we investigated the role of S1PR1 in HIV dissemination. We assessed the effects of treatment with FTY720 (fingolimod), a potent S1PR1 functional antagonist (20), alone or in combination with the CCR7 mAb (Figure 3A), as FTY720 has been shown to partially inhibit T cell egress alone and modestly add to CCR7 deficiency in blocking T cell egress in a model of skin inflammation (10). At 14 dpi, 0 of 12 FTY720 and 0 of 4 FTY720 + CCR7 mAb-treated mice showed positive viremia, while 7 of 8 control mice were viremic (Figure 3B). Aside from 1 mouse in the FTY720 + CCR7 mAb group, all mice had similar levels of vRNA in CVT (Figure 3C), demonstrating that the treatments did not interfere with local infection. This is consistent with prior studies that found that FTY720 treatment does not impact HIV infection in vitro or during established viremia in vivo (16). All PBS-treated mice had detectable vRNA in the cLNs and axillary LNs (aLNs), and 6 of 8 mice were positive in the mLNs and gut. Ten of 12 FTY720-treated mice were positive in either or both cLNs and aLNs. However, the mean viral loads in LNs of FTY720treated mice were lower than in PBS-treated mice, suggesting that FTY720 interfered with viral dissemination to these LNs despite not blocking it completely (Figure 3C). Similar results were obtained for FTY720 + CCR7 mAb-treated mice, showing that targeting CCR7 in addition to S1PR1 did not further impede viral dissemination (Figure 3C). These results were confirmed by vDNA analysis (Supplemental Figure 9).

S1PR1 signaling is also required for T cells to exit LNs and enter the blood (21). Accordingly, FTY720 treatment induced pronounced lymphopenia in the peripheral blood of both FTY720-treated and FTY720 + CCR7 mAb-treated groups of humanized BLT mice compared with controls (Figure 3D and Supplemental Figure 10). As expected, by day 14, FTY720-treated BLT mice demonstrated higher T cell numbers in LNs and decreased numbers in the spleen compared with PBS-treated mice, due to retention of lymphocytes in the LNs (Figure $3 \mathrm{E}$ and Supplemental Figure 10). In contrast, T cell numbers in the LNs and spleen of FTY720 + CCR7 mAb-treated mice were comparable or lower than in PBS-treated mice, due to the combined effects of FTY720 retaining T cells in the LNs and CCR7 mAb blocking the homing of naive $\mathrm{T}$ cells $\left(\mathrm{CD} 45 \mathrm{RA}^{+} \mathrm{CD} 62 \mathrm{~L}^{+}\right.$) into the LNs (Figure 3F and Supplemental Figure 10).

FTY720's inhibition of lymphocyte egress was associated with an inhibition of HIV viremia and spread to peripheral tissue (Figure 3, B and C). Only 1 of 12 FTY720-treated mice had detectable virus in mLNs, gut (Figure 3C), and lung (data not shown). This mouse also showed borderline viremia, whereas all other FTY720-treated mice had no detectable HIV in the plasma (Figure 3B). One of 4 FTY720 + CCR7 mAb-treated mice had detectable virus in mLNs, but this mouse did not have virus in the gut or the plasma. These data suggest that HIV is also actively carried out of LNs and into peripheral tissue and blood by migrating lymphocytes.

Pertussis toxin treatment restrains dissemination from the CVT. As FTY720 treatment only partially blocked viral spread to the LNs, we used pertussis toxin (PTX), an inhibitor of all Gai-coupled chemoattractant receptors, to determine whether additional chemoattractant receptors are involved in the trafficking of HIV-infected or HIV-bearing cells from the CVT to the LNs and contribute to viral dissemination.

PTX is composed of 1 enzymatic subunit (A) that inactivates Gai, which is required for chemoattractant receptor-mediated cell migration, and 5 receptor-binding $\mathrm{B}$ subunits $\left(\mathrm{AB}_{5}\right)(22)$. PTX treatment efficiently inhibited the trafficking of central memory-like murine CD4 ${ }^{+} \mathrm{T}$ cells to the draining LNs following IVAG injection (Supplemental Figure 11). Gai signaling is not required for R5-tropic HIV entry and replication in cells (23-27). However, some studies have reported that PTX, as well as its catalytically 
inactive B subunit (PTB), can interfere with HIV infection (28-30). We found that treatment with PTX or PTB starting 3 hpi had no impact on HIV $_{\text {JRCSF }}$ infection of MAGI cells or central memory-like human $\mathrm{CD}^{+} \mathrm{T}$ cells in vitro (Supplemental Figure 12, A and B). BLT mice were therefore treated with IVAG applications of PTX, PTB (to control for potential non-Gai affects of PTX), or PBS starting 3 hpi (experimental design shown schematically in Figure 4A).

At 14 dpi, 8 of 9 PTX-treated mice had no detectable viremia, while all PBS- $(n=7)$ and PTB-treated $(n=6)$ mice were viremic (Figure 4B). Five PBS-, 6 PTX-, and 3 PTB-treated mice were also subjected to a third week of treatment to determine whether inhibition of viremia could be maintained in PTX-treated mice. At 21 dpi, 3 of 6 PTX-treated mice remained aviremic (Figure 4B). The presence of vRNA was determined at $14 \mathrm{dpi}$ (Figure 4C, triangles) and $21 \mathrm{dpi}$ (Figure 4C, circles). All mice but 1 had similar levels of vRNA in the CVT, demonstrating that PTX or PTB treatment did not interfere with the acquisition of a local infection. The absence of vRNA in the CVT of the 1 PTX-treated mouse was likely a technical issue, as vDNA was found in the CVT of this mouse (orange in Supplemental Figure 13). None of the PTXtreated mice had detectable vRNA in tissues other than the CVT at $14 \mathrm{dpi}$. The absence of tissue vRNA was maintained at $21 \mathrm{dpi}$ in the 3 PTX-treated mice that were aviremic. All PBS- and PTB-treated mice were positive in the LNs and gut, except for 1 PBS- and 1 PTB-treated mouse that had no detectable vRNA in the aLNs. This might have been due to the small size of the aLNs in these BLT-NSG mice, where lower amounts of RNA were obtained than the other samples. PTX treatment had no impact on cell viability in the CVT and LNs or on human T cell numbers in the tissues (Supplemental Figure 12, C-E). Thus, vaginal PTX treatment markedly delayed dissemination of HIV to LNs by restraining HIV within the CVT. These results were confirmed by vDNA analysis (Supplemental Figure 13).

\section{Discussion}

Our data demonstrate that chemoattractant receptor-mediated leukocyte migration plays a critical role in HIV spread from the CVT to the LNs. We have found that viral dissemination was partially dependent on S1PR1-mediated leukocyte migration but was independent of CCR7-mediated cell trafficking. However, viral dissemination was completely inhibited by PTX in most mice, suggesting that other chemoattractant receptors not yet identified participate in this process. Our results are consistent with previous reports showing that in inflammation there is CCR7-independent $\mathrm{T}$ cell egress that is partially dependent on S1PR1 and completely inhibited by PTX $(10,11)$. Furthermore, trafficking of Yersinia pestis-infected mononuclear phagocytes from peripheral tissue to secondary lymphoid organs to form buboes has been shown to be dependent in large part on S1PR1 (19). However, it is also possible that the decrease in the viral load observed in aLNs and cLNs of FTY720-treated BLT-NSG mice following HIV IVAG infection is due to impaired trafficking of HIV-transporting cells from the draining iLNs to the other LNs and not directly from the CVT to these LNs. Unfortunately, the iLNs of BLT-NSG mice were too small to be analyzed to formally test this alternative hypothesis. We also cannot completely rule out the possibility that the CCR7 pathway might contribute to the egress of infected cells or cells carrying HIV. It is possible that the mAb treatment did not completely block CCR7 function in the CVT, allowing enough cells to leave the tissue and spread the infection to the lymphoid compartment. However, we found that CCR7 on T cells recovered from tissues was blocked following in vivo CCR7 mAb treatment.

As mentioned, pretreatment of cells with PTX or PTB has been reported to inhibit HIV infection of activated $\mathrm{CD}^{+} \mathrm{T}$ cells and reporter cell lines in vitro $(28,31)$. Similarly, treatment of cervical explants prior to HIV exposure has been reported to suppress infection (29). The antiviral activity of PTB has also been previously reported in vivo in immunodeficient mice reconstituted with human peripheral blood mononuclear cells (30). In these mice, i.p. injection of $50 \mathrm{ng}$ of PTB, starting 2 hours prior to HIV inoculation i.p., was found to protect against HIV acquisition. Curiously and inexplicably, the antiviral effect of PTB was not seen when $500 \mathrm{ng}$ was given. In our experiments, IVAG daily treatment was started 3 hpi with $500 \mathrm{ng}$ of PTX or PTB, which corresponds to the dose that was reported to be ineffective against infection in huPBL mice (30). In our experiments, PTB treatment had no effect on plasma viremia or tissue viral loads and PTB-treated mice were comparable to PBS-treated mice. Furthermore, similar levels of HIV RNA were found in the CVT of mice across treatment groups, demonstrating that PTX treatment did not impact local infection, supporting the conclusion that the effect of PTX on viral dissemination was due to the inhibition of leukocyte trafficking and not due to a direct antiviral effect of the toxin. 
Our data also suggest that HIV is actively carried into peripheral tissue and blood by migrating lymphocytes, as the infection can be confined to the LNs by blocking S1PR1-mediated lymphocyte egress from LNs. This is consistent with our previous observation that FTY720 prevented HIV from leaving the dLNs following subcutaneous cheek injection in BLT-NSG mice (16). In addition, following IVAG infection, a clear delay was observed when HIV could be recovered from the iLNs and cLNs compared with the mLNs, gut, and blood. Consistent with this pattern of dissemination, S1PR1 blockade protected mLNs and gut from infection. Lymphocyte trafficking into the gut depends on CCR9 and $\alpha 4 \beta 7$ (32), while lymphocyte entry into mLNs can also be mediated by CCR7, CXCR4, and L-selectin (33). Treatment of NHPs during acute SIV infection with an anti- $\alpha 4 \beta 7 \mathrm{mAb}$ was found to decrease gut and plasma viral loads (34), suggesting that lymphocyte trafficking is also important for disseminating SIV infection to the gut in NHPs. Taken together, these results suggest that the spread of infection from the CVT to the draining LNs occurs via infected or virus-carrying cells that enter the afferent lymphatics draining the CVT, and from there can rapidly spread to other LNs, such as the cLNs and aLNs. In contrast, the spread of HIV to the mLNs occurs later and coincides with the infection reaching the gut, suggesting that HIV spreads to mLNs via the blood or from afferent lymph draining the gut. Passage through LNs could be a key step for viral dissemination by allowing a rapid expansion of infection due to the large number of target cells present in close proximity in the LNs but could also be required for imprinting infected T cells with tissue-homing receptors (e.g., CCR9 and $\alpha 4 \beta 7$ ), enabling infected T cells to home to certain peripheral tissue, such as the gut.

Our results in FTY720-treated mice differ from those reported with low-dose FTY720 treatment of NHPs, which was not effective at preventing viremia following vaginal infection with an SIV/HIV (SHIV) chimeric virus (35). However, FTY720 treatment of NHPs in that study decreased peripheral blood lymphocyte numbers only by 2-fold, compared with the 100 -fold decease in our study, suggesting that differing effectiveness of FTY720 treatment in these studies could explain the differences in viremia outcomes. In addition, differences between HIV and SIV/SHIV that influence, among others things, host cell permissiveness for replication (36), could also be contributory, highlighting the importance of the BLT model to study HIV transmission and dissemination. The PTX result reinforces our inference that the decreased viral load observed in the LNs of FTY720-treated mice was due to the blockade of S1PR1-dependent leukocyte trafficking. The fact that CCR7 blockade and FTY720 treatment alone or together did not completely block dissemination of infection from the CVT to the LNs suggests that other chemoattractant receptors not yet identified also participate in this process.

Similar to the experiments describing SIV dissemination in NHP (2, 37-40), we used a single high-titer virus in progesterone-treated BLT mice to allow a reliable and robust infection needed to compare the effect of the different treatments. These conditions might not exactly mirror a physiologic vaginal HIV exposure, as they facilitate HIV transmission across the vaginal epithelium (41). However, we believe that this model is relevant to study mechanisms of viral dissemination after viral acquisition in the CVT. The target cells that HIV will encounter once in the vaginal mucosa are indeed comparable in BLT mice and in humans $(12,15,42,43)$.

While the NHP model of SIV/SHIV infection allows for the study of early events of SIV infection, it does not allow for the study of HIV infection. The humanized mouse model is therefore an important adjunct model to study HIV infection in vivo. Our data show that HIV dissemination in BLT mice reproduces some aspects of the paradigm proposed for SIV infection of NHPs $(2,38,44)$, where SIV has been shown to spread to the LNs following infection of the FGT before dissemination to distant tissues and detection in blood. A recent publication (39) showed that, in a few cases, SIV RNA could be found in distant tissues as early as $24 \mathrm{hpi}$. This suggests that rapid SIV dissemination that bypasses the need for local infection and spread to the LNs can occur occasionally. In our PTX experiments, 1 of 9 PTX-treated mice showed positive viremia at $14 \mathrm{dpi}$, suggesting that in rare instances, HIV dissemination might occur in a cell-free manner.

The increase in cytokines observed in the CVT and LNs of BLT mice following IVAG HIV infection also resembles the immune response reported in the FGT and draining LNs in NHPs following SIV IVAG application $(38,39,44)$. In NHPs, increased staining for the chemokines CCL3, CCL20, CXCL8, and CXCL10 was reported in the FGT starting at $1 \mathrm{dpi}(38,44)$. An interferon-stimulated gene signature was also reported in infected tissue as early as $1 \mathrm{dpi}(39)$. In our studies, we found the induction of the genes encoding the interferon-inducible chemokines CXCL9 and CXCL10, as well as TNF- $\alpha$, in the CVT 1 dpi and in the LNs starting at $2 \mathrm{dpi}$. In addition, immune cell clusters containing $\mathrm{CD} 4^{+}$target $\mathrm{T}$ cells were observed in the CVT of BLT mice at 2 dpi as has been described in the FGT of NHPs (38). 
Humanized BLT mice are not a perfect model of HIV infection, as they are prone to immune activation and graft-versus-host disease (GvHD), and have other limitations that have been discussed in several recent reviews addressing the use of humanized mouse models of HIV infection (45-48). Nevertheless, humanized mouse models are the only models that allow the study of the dynamics of HIV infection in vivo and BLT mice represent, to date, the most complete humanized mouse model. Our results demonstrate that HIV dissemination in humanized BLT mice mirrors the previously described paradigm of SIV dissemination in NHPs. In addition, we demonstrate that interfering with cell trafficking can restrict HIV infection to the FGT. This will prolong the therapeutic window during which the virus can be targeted before it spreads to the lymphoid compartment and peripheral tissue where it induces massive CD4 ${ }^{+} \mathrm{T}$ cell depletion (49) and establishes a latent reservoir that is recalcitrant to eradication with current treatments. Finally, our results emphasize the potential importance of therapeutic strategies that target the migration of infected and virusbearing cells, as they serve as Trojan horses to disseminate the virus following vaginal acquisition.

\section{Methods}

Generation of humanized BLT mice. Female BLT-NOD-scid (NS) or BLT-NOD-scid IL2Rg ${ }^{-1}$ (NSG) mice (The Jackson Laboratory) were housed in a pathogen-free facility at Massachusetts General Hospital and reconstituted with human tissue as described (13). Briefly, sublethally irradiated mice were transplanted under the kidney capsule with $1-\mathrm{mm}^{3}$ fragments of human fetal liver and thymus, and injected intravenously with purified $\mathrm{CD}_{3} 4^{+}$human fetal liver cells to generate humanized BLT mice. Human immune cell reconstitution was monitored 14-18 weeks after surgery and considered sufficient if more than $40 \%$ of lymphocytes were human $\mathrm{CD}_{4} 5^{+}$, of which more than $30 \%$ were $\mathrm{CD}^{+}$, and there were at least $200 \mathrm{CD}^{+} \mathrm{T}$ cells $/ \mu 1$ in peripheral blood. The HLA locus of each donor was genotyped using PCR-sequence-specific oligonucleotide probing (PCR-SSOP) and PCR-sequence-based typing (PCR-SBT) (Supplemental Table 1). Clinical signs of $\mathrm{GvHD}$, such as conjunctivitis, blepharitis, alopecia, dermatitis, and weight loss were monitored for each mouse biweekly. BLT mice showing clinical signs of GvHD at any time during the experiments were excluded. Of note, clinical signs of GvHD in skin and eyes were found to precede histological signs of GvHD in the female genital tract and gut, including infiltration of human $\mathrm{CD} 45^{+}$cells (data not shown). Once it was determined that the pattern and kinetics of HIV dissemination were similar in BLT-NS and BLT-NSG mice, BLT-NSG mice were then preferentially used since they are more easily reconstituted with human immune cells and therefore allow higher numbers of reconstituted animals per batch of human tissue.

$H I V$ infection of humanized BLT mice. Viral stocks of the $\mathrm{HIV}_{\text {JRCSF }}$ molecular clone were produced through transfection of human embryonic kidney (HEK) 293T cells and titered as described (30). Mice were infected with atraumatic IVAG application of $1 \times 10^{5} \mathrm{TCID}_{50} \mathrm{HIV}_{\text {JRCSF }}$ in 10 to $20 \mu 1 \mathrm{PBS}$ as previously described (12). Mice were treated 5 days prior to challenge with a subcutaneous injection of $200 \mu \mathrm{g}$ of progesterone (Depo-Provera medroxyprogesterone acetate, Pfizer) in a total volume of $100 \mu 1$ of PBS.

Pharmacological and antibody treatment. A neutralizing fully humanized non-cell-depleting anti-human CCR7 IgG1 monoclonal antibody without N-linked glycosylation sites was provided by Amgen. BLT mice were treated with $100 \mu$ g of CCR7 mAb i.p. in combination with $10 \mathrm{mg}$ (i.p.) of pooled human $\gamma$-globulins (Sigma-Aldrich) and $200 \mu \mathrm{g}$ (i.p.) 2.4G2 (Fc receptor blocker, provided by Amgen) every third day starting 1 day prior to HIV IVAG and $5 \mu \mathrm{g}(10 \mu \mathrm{l})$ CCR7 mAb IVAG daily starting 3 hours after HIV infection. Control mice received $10 \mathrm{mg}$ of human $\gamma$-globulins and $200 \mu \mathrm{g}$ 2.4G2 (rat-IgG2b-anti-muFcRII) i.p. and equivalent volumes of human CCR7 mAb in PBS i.p. and IVAG. FTY720 at $1 \mathrm{mg} / \mathrm{kg} / \mathrm{body}$ weight (Cayman Chemicals) or vehicle was injected i.p. daily starting 4 days to 3 hours before HIV infection to induce peripheral blood lymphopenia and $0.5 \mu \mathrm{g}(10 \mu \mathrm{l})$ IVAG daily starting 3 hours after HIV infection. PTX (Sigma-Aldrich, $4.2 \mathrm{nM}, 0.5 \mu \mathrm{g}$ in $10 \mu \mathrm{PBS}$ ), PTB (Sigma-Aldrich, $4.2 \mathrm{nM}, 0.4 \mu \mathrm{g}$ in $10 \mu 1 \mathrm{PBS}$ ), or PBS $(10 \mu \mathrm{l})$ was applied IVAG daily starting 3 hours after HIV infection. IVAG treatment was started 3 hours after HIV exposure to avoid diluting the inoculum and to allow the infection to seed the CVT.

Plasma viral load. BLT mice were bled from the superficial temporal vein and plasma was stored at $-80^{\circ} \mathrm{C}$. vRNA was extracted from 50 to $70 \mu$ plasma using a QIAamp Viral RNA Kit (Qiagen). Reverse transcriptase quantitative PCR (RT-qPCR) was performed using HIV gag-specific primers (SK145 forward, AGTGGGGGGACATCAAGCAGCCATGCAAAT and SK431 reverse, TGCTATGTCACTTCCCCTTGGTTCTCT) and the QuantiTect SYBR Green RT-PCR Kit (Qiagen) on the Lightcycler 480-II (Roche) as previously described (50). Plasma from uninfected BLT mice was used to determine background signal. The limit of detection of the assay was 3 RNA copies/ $\mu 1$. 
Tissue viral load. Mice were euthanized at indicated time points and tissues were harvested in RNAlater solution (Ambion) and incubated at $4^{\circ} \mathrm{C}$ overnight before being stored at $-80^{\circ} \mathrm{C}$. Tissue was homogenized using gentle MACS Dissociator (Miltenyi Biotec) in TRIzol solution (Thermo Fisher Scientific) and RNA was extracted following the TRIzol manufacturer's protocol. DNA was extracted from TRIzol solution using back extraction buffer and following the supplier's protocol. Polyacryl Carrier (5 $\mu$ l) (Molecular Research Center) was added during the precipitation for increased yield. A second precipitation step using sodium acetate was added to increase DNA purity. Quantification of vRNA was performed as described above. Quantification of vDNA was performed using the qPCR SYBR Green PCR Kit on the Lightcycler 480-II. Viral loads were normalized to total tissue RNA and DNA. Background signal was measured for each tissue using 3-5 uninfected animals. The mean signal value obtained from uninfected tissue $\pm 2 \mathrm{SD}$ was used as the detection limit. For some of the gut samples, nonspecific primer binding was observed for vDNA PCR, which resulted in the exclusion of those samples from the vDNA results in the CCR7 and FTY720 experiments.

Flow cytometry. Human lymphocyte characterization was performed on an LSR II (BD Biosciences) and analyzed using FlowJo software (Tree Star). CVT from BLT mice and human cervical tissues were minced using the gentle MACS Dissociator and incubated in medium containing Liberase $(2.5 \mathrm{mg} / \mathrm{ml})$ and DNAse $(0.03 \mathrm{mg} / \mathrm{ml}$ ) (both Sigma-Aldrich). Human cervical tissue was obtained from excess surgical tissue from healthy human donors through the Ragon Institute of MGH, MIT and Harvard Tissue Platform (Cambridge, Massachusetts, USA). Spleen and LNs from BLT mice were minced using scissors. Processed tissues were passed through a $40-\mu \mathrm{m}$ mesh strainer to obtain single-cell suspensions. Cells were stained using directly labeled anti-human monoclonal antibodies (Biolegend): CD45 (HI30), CD3 (HIT3a), CD4 (OKT4), CD8 (RPA-T8), CCR7 (TG8), CD45RA (HI100), and CD62L (DREG-56). Dead cells were excluded using LIVE/DEAD Fixable Stain (Life Technologies). Cells were fixed with $\%$ paraformaldehyde after staining. Whole blood (15-50 $\mu \mathrm{l})$ was directly stained with antibodies and treated with FACS lysing solution (BD Biosciences) before analysis. Since human CCR7 mAb blocked subsequent CCR7 staining (Supplemental Figures 6 and 7), CD62L was used as marker to define human T cell subsets (51) in the experiments involving CCR7 mAb treatment. Human T cell number and phenotype in LNs of mice treated with FTY720 and/or CCR7 mAb were determined using cervical and axillary LNs.

Statistics. Unpaired Student's $t$ test or Kruskal-Wallis test with Dunn's post hoc test was used to test for differences in mean values. Fisher's exact test was used to test for differences in the number of infected and uninfected animals of in vivo experiments. All $P$ values are 2-sided and $P$ less than 0.5 was considered statistically significant. Statistical analysis and graphing were performed using GraphPad Prism. Columns represent mean \pm SD.

Study approval. All animal experiments have been reviewed and approved by the Massachusetts General Hospital IACUC. Human cervical tissue was obtained from excess surgical tissue following an IRB-approved protocol at Massachusetts General Hospital.

\section{Author contributions}

MD, TTM, and SJ performed all experiments. VDV generated humanized BLT mice. MAG, JL, AMT, and TRM contributed to the overall study design. MD and ADL designed the experiments and wrote the manuscript.

\section{Acknowledgments}

We would like to thank Robert Anthony for his expertise and assistance in confirming the absence of glycosylation of the CCR7 mAb and Jeffrey Pear, Douglas Kwon, and the Ragon Institute Tissue Platform for their help in obtaining human tissue samples. This work was supported by grants from the NIH (P01AI112521, R01AI097052, and R01DA036298), the Harvard University Center for AIDS Research (P30AI060354), and the Ragon Institute of MGH, MIT and Harvard.

Address correspondence to: Andrew D. Luster, Center for Immunology and Inflammatory Diseases, Division of Rheumatology, Allergy and Immunology, 149 Thirteenth Street, Charlestown, Massachusetts 02129, USA. Phone: 617.726.5710; E-mail: aluster@mgh.harvard.edu.

TTM's present address is: Department of Immunology and Medical Microbiology, University of Manitoba, Winnipeg, Canada. 
MAG's present address is: Benaroya Research Institute, Seattle, Washington, USA.

1. UNAIDS. The Gap Report. http://www.unaids.org/en/resources/campaigns/2014/2014gapreport/gapreport. Published July 14, 2014. Accessed March 3, 2017.

2. Miller CJ, et al. Propagation and dissemination of infection after vaginal transmission of simian immunodeficiency virus. J Virol. 2005;79(14):9217-9227.

3. Ma ZM, Dutra J, Fritts L, Miller CJ. Lymphatic dissemination of simian immunodeficiency virus after penile inoculation. J Virol. 2016;90(8):4093-4104.

4. McMichael AJ, Borrow P, Tomaras GD, Goonetilleke N, Haynes BF. The immune response during acute HIV-1 infection: clues for vaccine development. Nat Rev Immunol. 2010;10(1):11-23.

5. Dale BM, Alvarez RA, Chen BK. Mechanisms of enhanced HIV spread through T-cell virological synapses. Immunol Rev. 2013;251(1):113-124.

6. Hladik F, et al. Initial events in establishing vaginal entry and infection by human immunodeficiency virus type-1. Immunity. 2007;26(2):257-270

7. Bromley SK, Thomas SY, Luster AD. Chemokine receptor CCR7 guides $\mathrm{T}$ cell exit from peripheral tissues and entry into afferent lymphatics. Nat Immunol. 2005;6(9):895-901.

8. Debes GF, et al. Chemokine receptor CCR7 required for T lymphocyte exit from peripheral tissues. Nat Immunol. 2005;6(9):889-894.

9. Förster R, et al. CCR7 coordinates the primary immune response by establishing functional microenvironments in secondary lymphoid organs. Cell. 1999;99(1):23-33.

10. Brown MN, et al. Chemoattractant receptors and lymphocyte egress from extralymphoid tissue: changing requirements during the course of inflammation. J Immunol. 2010;185(8):4873-4882.

11. Ledgerwood LG, et al. The sphingosine 1-phosphate receptor 1 causes tissue retention by inhibiting the entry of peripheral tissue T lymphocytes into afferent lymphatics. Nat Immunol. 2008;9(1):42-53.

12. Denton PW, et al. Antiretroviral pre-exposure prophylaxis prevents vaginal transmission of HIV-1 in humanized BLT mice. PLoS Med. 2008;5(1):e16.

13. Brainard DM, et al. Induction of robust cellular and humoral virus-specific adaptive immune responses in human immunodeficiency virus-infected humanized BLT mice. J Virol. 2009;83(14):7305-7321.

14. Wheeler LA, et al. Inhibition of HIV transmission in human cervicovaginal explants and humanized mice using CD4 aptamersiRNA chimeras. J Clin Invest. 2011;121(6):2401-2412.

15. Olesen R, et al. ART influences HIV persistence in the female reproductive tract and cervicovaginal secretions. J Clin Invest. 2016;126(3):892-904

16. Murooka TT, et al. HIV-infected T cells are migratory vehicles for viral dissemination. Nature. 2012;490(7419):283-287.

17. Albert H, Collin M, Dudziak D, Ravetch JV, Nimmerjahn F. In vivo enzymatic modulation of IgG glycosylation inhibits autoimmune disease in an IgG subclass-dependent manner. Proc Natl Acad Sci USA. 2008;105(39):15005-15009.

18. Seung E, Tager AM. Humoral immunity in humanized mice: a work in progress. J Infect Dis. 2013;208 Suppl 2:S155-S159.

19. St John AL, et al. S1P-dependent trafficking of intracellular yersinia pestis through lymph nodes establishes buboes and systemic infection. Immunity. 2014;41(3):440-450.

20. Brinkmann V, et al. The immune modulator FTY720 targets sphingosine 1-phosphate receptors. J Biol Chem. 2002;277(24):21453-21457.

21. Cyster JG, Schwab SR. Sphingosine-1-phosphate and lymphocyte egress from lymphoid organs. Annu Rev Immunol. 2012;30:69-94.

22. Spangrude GJ, Sacchi F, Hill HR, Van Epps DE, Daynes RA. Inhibition of lymphocyte and neutrophil chemotaxis by pertussis toxin. J Immunol. 1985;135(6):4135-4143.

23. Cocchi F, DeVico AL, Garzino-Demo A, Cara A, Gallo RC, Lusso P. The V3 domain of the HIV-1 gp120 envelope glycoprotein is critical for chemokine-mediated blockade of infection. Nat Med. 1996;2(11):1244-1247.

24. Farzan M, et al. HIV-1 entry and macrophage inflammatory protein-1beta-mediated signaling are independent functions of the chemokine receptor CCR5. J Biol Chem. 1997;272(11):6854-6857.

25. Amara A, et al. G protein-dependent CCR5 signaling is not required for efficient infection of primary $\mathrm{T}$ lymphocytes and macrophages by R5 human immunodeficiency virus type 1 isolates. $J$ Virol. 2003;77(4):2550-2558.

26. Harmon B, Ratner L. Induction of the Galpha(q) signaling cascade by the human immunodeficiency virus envelope is required for virus entry. J Virol. 2008;82(18):9191-9205.

27. Colin P, et al. HIV-1 exploits CCR5 conformational heterogeneity to escape inhibition by chemokines. Proc Natl Acad Sci USA. 2013;110(23):9475-9480.

28. Alfano M, Pushkarsky T, Poli G, Bukrinsky M. The B-oligomer of pertussis toxin inhibits human immunodeficiency virus type 1 replication at multiple stages. J Virol. 2000;74(18):8767-8770.

29. Hu Q, Younson J, Griffin GE, Kelly C, Shattock RJ. Pertussis toxin and its binding unit inhibit HIV-1 infection of human cervical tissue and macrophages involving a CD14 pathway. J Infect Dis. 2006;194(11):1547-1556.

30. Lapenta C, et al. Pertussis toxin B-oligomer inhibits HIV infection and replication in hu-PBL-SCID mice. Int Immunol. 2005;17(4):469-475.

31. Alfano M, Schmidtmayerova H, Amella CA, Pushkarsky T, Bukrinsky M. The B-oligomer of pertussis toxin deactivates CC chemokine receptor 5 and blocks entry of M-tropic HIV-1 strains. J Exp Med. 1999;190(5):597-605.

32. Zabel BA, et al. Human G protein-coupled receptor GPR-9-6/CC chemokine receptor 9 is selectively expressed on intestinal homing $\mathrm{T}$ lymphocytes, mucosal lymphocytes, and thymocytes and is required for thymus-expressed chemokine-mediated chemotaxis. J Exp Med. 1999;190(9):1241-1256.

33. Habtezion A, Nguyen LP, Hadeiba H, Butcher EC. Leukocyte trafficking to the small intestine and colon. Gastroenterology. 2016;150(2):340-354. 
34. Ansari AA, et al. Blocking of $\alpha 4 \beta 7$ gut-homing integrin during acute infection leads to decreased plasma and gastrointestinal tissue viral loads in simian immunodeficiency virus-infected rhesus macaques. J Immunol. 2011;186(2):1044-1059.

35. Morris M, et al. Preclinical evaluation of the immunomodulatory lymphocyte trafficking drug FTY720 for HIV prevention in the female genital mucosa of macaques. J Med Primatol. 2014;43(5):370-373.

36. Manel N, Hogstad B, Wang Y, Levy DE, Unutmaz D, Littman DR. A cryptic sensor for HIV-1 activates antiviral innate immunity in dendritic cells. Nature. 2010;467(7312):214-217.

37. Li Q, et al. Peak SIV replication in resting memory CD4+ $\mathrm{T}$ cells depletes gut lamina propria $\mathrm{CD} 4^{+} \mathrm{T}$ cells. Nature. 2005;434(7037):1148-1152.

38. Shang L, et al. Epithelium-innate immune cell axis in mucosal responses to SIV. Mucosal Immunol. 2017;10(2): 508-519.

39. Barouch DH, et al. Rapid Inflammasome activation following mucosal SIV infection of rhesus monkeys. Cell. 2016;165(3):656-667.

40. Liu J, et al. Antibody-mediated protection against SHIV challenge includes systemic clearance of distal virus. Science. 2016;353(6303):1045-1049.

41. Khanna KV, et al. Vaginal transmission of cell-associated HIV-1 in the mouse is blocked by a topical, membrane-modifying agent. J Clin Invest. 2002;109(2):205-211.

42. Johansson EL, Rudin A, Wassén L, Holmgren J. Distribution of lymphocytes and adhesion molecules in human cervix and vagina. Immunology. 1999;96(2):272-277.

43. Pudney J, Quayle AJ, Anderson DJ. Immunological microenvironments in the human vagina and cervix: mediators of cellular immunity are concentrated in the cervical transformation zone. Biol Reprod. 2005;73(6):1253-1263.

44. Li Q, et al. Glycerol monolaurate prevents mucosal SIV transmission. Nature. 2009;458(7241):1034-1038.

45. Garcia JV. In vivo platforms for analysis of HIV persistence and eradication. J Clin Invest. 2016;126(2):424-431.

46. Akkina R, et al. Improvements and limitations of humanized mouse models for HIV research: NIH/NIAID "Meet the Experts" 2015 workshop summary. AIDS Res Hum Retroviruses. 2016;32(2):109-119.

47. Deruaz M, Luster AD. BLT humanized mice as model to study HIV vaginal transmission. J Infect Dis. 2013;208 Suppl 2:S131-S136.

48. Karpel ME, Boutwell CL, Allen TM. BLT humanized mice as a small animal model of HIV infection. Curr Opin Virol. $2015 ; 13: 75-80$.

49. Brenchley JM, et al. CD4 ${ }^{+} \mathrm{T}$ cell depletion during all stages of HIV disease occurs predominantly in the gastrointestinal tract. JExp Med. 2004;200(6):749-759

50. Boutwell CL, Rowley CF, Essex M. Reduced viral replication capacity of human immunodeficiency virus type 1 subtype C caused by cytotoxic-T-lymphocyte escape mutations in HLA-B57 epitopes of capsid protein. J Virol. 2009;83(6):2460-2468

51. Sallusto F, Lenig D, Förster R, Lipp M, Lanzavecchia A. Pillars article: two subsets of memory T lymphocytes with distinct homing potentials and effector functions. Nature. 1999. 401: 708-712. J Immunol. 2014;192(3):840-844. 